\title{
Fast scanning using piezoelectric tube nanopositioners: A negative imaginary approach.
}

\author{
B. Bhikkaji, S. O. R. Moheimani, Senior Member, IEEE,
}

\begin{abstract}
Most commercially available Atomic Force Microscopes (AFMs) use piezoelectric tube nano-positioners for scanning. Current scanning frequencies are less than $0.01 f_{r}$, where $f_{r}$ is the frequency of the first resonant mode of the piezoelectric tube used. An improvement in the scanning rates without losing the nano-scale precision is desired. Here, a prototype of the scanning unit of an AFM is considered. The dynamics of the piezo tube, used in the prototype, is approximated by a model that satisfies the negative imaginary property. The resonant mode that hampers the fast scanning is identified from the model and damped using a feedback control technique known as the Integral Resonant Control (IRC). The piezoelectric tube is then actuated to have fast and accurate scans.
\end{abstract}

\section{INTRODUCTION}

Scanning Tunneling Microscopes (STMs) and Atomic Force Microscopes (AFMs), when used at extreme magnifications, are capable of generating topographical maps of solid surfaces at micro to atomic resolution. In many STMs and AFMs a probe is placed in close proximity, typically a few nanometers, to the material surface for which a topographical map is desired. The given material surface is scanned by moving the sample in a raster pattern, so that the probe interacts with the entire region of interest [6]. In general, scanning is performed by placing the sample on top of a piezoelectric tube and actuating the tube in a raster pattern.

Dynamics of piezoelectric tubes can be well approximated, under certain experimental conditions, by linear models, see [9], [16], [18]. The linear models normally reveal the presence of lightly damped resonance modes which make the piezoelectric tubes susceptible to mechanical vibrations. Non-linear phenomenon such as creep and hysteresis can also be observed when actuating the tube using low frequency and high amplitude inputs respectively, [10]. In such scenarios the linear approximations become inadequate. Current scanning frequencies are limited to less than $0.01 f_{r}$, where $f_{r}$ is the first resonance frequency of the piezoelectric tube. In this paper the piezoelectric tube is scanned at a frequencies comparable to $0.1 f_{r}$. The main impediments to such scans are the presence of mechanical vibrations and hysteresis.

Slow scans are fast becoming a bane to the users of AFMs. Slow scans, though accurate, lead to inordinate waiting periods before an image can be obtained. A good case for the

B. Bhikkaji, Dept. of Elec. Engg, Indian Institute of Technology Madras, Chennai-36, India, email: Bharath.Bhikkaji@iitm.ac.in and S. O. R. Moheimani, School of Electrical Engineering and Computer Science, The University of Newcastle, Callaghan, NSW 2308, Australia, e-mail: Reza.Moheimani@newcastle.edu.au. design of control systems to perform fast scans is presented in [2].

Mechanical vibrations can be compensated by designing suitable feedback controllers to damp the resonant modes. The opportunity to use feedback controllers has aroused the interest of many control engineers, [1], [9], [16]. Contemporary researchers have used standard techniques like PI, PID, LQG and $\mathrm{H}_{\infty}$ for designing controllers, [1], [9], [16], to damp the first resonant mode. Controllers like PI and the PID are easy to design and implement, however they are not robust to variations in plant parameters and also suffer from low tracking bandwidth [18]. Moreover, due to their structure, the chances of them exciting the unmodeled higher order modes of the tube are also high. More advanced controllers like LQG and $\mathrm{H}_{\infty}$ have strong theoretical foundations and their design methodologies are incorporated into standard softwares such as Matlab. Nevertheless, LQG and $\mathrm{H}_{\infty}$ controllers designed for large bandwidths may disturb the unmodeled modes. It is desirable to have controllers that are easy to implement, robust, have a roll-off in the high frequency regions and also damp the system.

Recently it came to light that systems with collocated sensor/actuator pairs satisfy the negative imaginary property, [12]. And under certain conditions such systems can be stabilized by controllers satisfying the same negative imaginary. Piezoelectric tubes do not have collocated sensor/actuator pairs but can be approximated by a model that satisfies the negative imaginary property. In [5] a new feedback scheme, called the integral resonant control (IRC) was introduced. IRC is relatively straightforward and easy to design and implement and rolls-off in the high frequency regions. The computational complexity involved in their design is also insignificant. In this paper this scheme is shown to have the negative imaginary property. An IRC controller is designed to damp the first resonant mode of a piezoelectric tube, resulting in a fast tracking of a high-bandwidth set point with nano-scale precision.

Nonlinearity in the form of hysteresis becomes visible when piezoelectric tubes are actuated using voltage signals of high amplitudes. A popular approach to compensate for hysteresis is to model it as a non-linear function, $H(\cdot)$, and then eliminate it by cascading its inverse $H^{-1}(\cdot)$ with piezoelectric tube actuator, see [7], [17]. Feedback control schemes have also been used to compensate for hysteresis in [18]. Since the late 1980s, it has been known that actuating piezoelectric transducers with current or charge sources rather than voltage sources significantly reduces hysteresis [15]. In fact it has been noted that a five-fold reduction in 
hysteresis can be achieved, [11], using charge sources.

Non-linear modeling of hysteresis has always been a tedious computational task, [13]. In cases where the hysteresis is rate dependent, as in the case of piezoelectric materials, current modeling schemes offer little help. Use of charge or current sources does not necessitate the modeling of hysteresis. However, herein, the problem lies with the instrumentation of the charge or current sources capable of driving highly capacitive loads, as they are not commercially available at present. Invariably, the only resort is to make a "home made" charge or current source. In [3] and [4], the authors have designed a DC-accurate charge source for the general purpose of exciting piezoelectric actuators without encountering hysteresis. In this work, this charge amplifier is used for applying signals to the piezoelectric tube in order to avoid hysteresis.

This paper is formatted as follows. In the next section, the control scheme presented in [5] is discussed from the perspective of using it for a piezoelectric tube. In Section 3, a piezoelectric tube of the type typically used in STMs and AFMs is considered and its resonance mode is identified and damped using the scheme presented in Section 2. The tube is also actuated in a raster pattern in Section 3.

\section{Integral ResonAnt CONTROL OF A PIEZOELECTRIC TUBE ACTUATOR.}

Piezoelectric tubes, like most other flexible structures, have an infinite number of resonant modes. However, from a computational perspective one has to consider only a finite number of modes. Most piezoelectric tubes used in STMs and AFMs would have their first resonant mode within a bandwidth of $1 \mathrm{KHz}^{1}$, while the other resonances would lie beyond $2 \mathrm{KHz}$, [9], [1], [19]. In order to actuate the piezoelectric tube at a rate of $0.1 f_{r}$, where $f_{r}$ is the first resonance frequency, it is enough to consider the first resonance alone.

The transfer-functions characterizing the dynamics of piezoelectric tubes, up to a bandwidth of $2 f_{r} \mathrm{~Hz}$, are typically of the form

$$
G(s)=\frac{\Gamma}{s^{2}+2 \zeta_{p} \omega_{p} s+\omega_{p}^{2}},
$$

where $\Gamma>0, \omega_{p}$ denotes the resonance frequency and $\zeta_{p}>0$ denotes the damping co-efficient, [9], [1].

Note that

$$
\mathrm{i}\left(G(\mathrm{i} \omega)-G^{*}(\mathrm{i} \omega)\right)=\frac{4 \zeta_{p} \omega_{p} \Gamma}{\left(\omega_{p}^{2}-\omega^{2}\right)^{2}+\left(2 \zeta_{p} \omega_{p} \omega\right)^{2}},
$$

is strictly positive. Hence, negative imaginary. In [5] a transfer function of the form (1) was considered. Adding an appropriate feed through term $d$ to (1),

$$
\bar{G}(s)=\frac{\Gamma}{s^{2}+2 \zeta_{p} \omega_{p} s+\omega_{p}^{2}}+d,
$$

\footnotetext{
${ }^{1}$ The actual resonance frequencies depend on the physical dimensions of the tube. It is possible to use small tubes that have very high resonance frequencies. However, most commercially available STMs and AFMs use relatively large tubes. This is needed to scan large areas, e.g., $100 \mu \mathrm{m} \times$ $100 \mu \mathrm{m}$. Such tubes have relatively large frequencies.
}

and wrapping an Integral controller $C(s)=\frac{K}{s}$ around $G(s)$, (3), in feed back configuration, see Figure 1, the authors were able to achieve significant damping. In order to explain the damping properties the authors first approximated (1) by

$$
G_{\text {approx }}(s)=\frac{\Gamma}{s^{2}+\omega_{p}^{2}} .
$$

Using the approximation they argued that for both $K$ and $d$ negative the closed loop stability can be guaranteed.

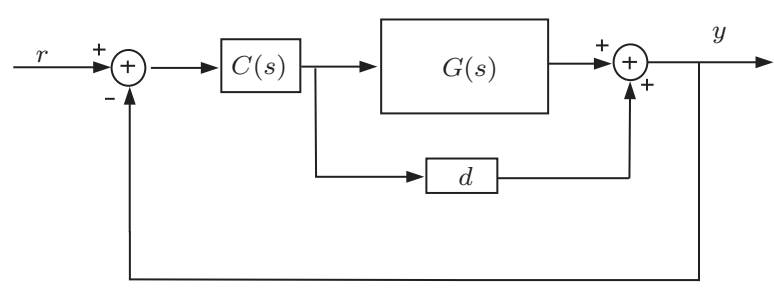

Fig. 1. Closed-loop system with the Integral controller $C(s)=\frac{K}{s}$ around $\bar{G}(s)$.

Note that wrapping an integral controller $C(s)=\frac{K}{s}$ around $\bar{G}(s)$ would lead to the closed-loop system, see Figure 1,

$$
\begin{aligned}
\bar{G}^{(c l)}(s) & =\frac{\bar{G}(s) \frac{K}{s}}{1+\frac{K}{s} \bar{G}(s)} \\
& =\frac{K d\left(s^{2}+\omega_{p}^{2}+\frac{\Gamma}{d}\right)}{s\left(s^{2}+\omega_{p}^{2}\right)+K d\left(s^{2}+\omega_{p}^{2}+\frac{\Gamma}{d}\right)} .
\end{aligned}
$$

It can be also checked that the closed-loop system corresponding to the plant input and output (i.e., with respect to $G(s))$ is given by

$$
G^{(c l)}(s)=\frac{G(s) \frac{K}{s}}{1+\frac{K}{s} \bar{G}(s)} .
$$

It can be noted that (6) boils down to

$$
\begin{aligned}
G^{(c l)}(s) & =\frac{G(s) \frac{K}{s}}{1+\frac{K}{s} \bar{G}(s)} \\
& =\frac{G(s) \frac{K}{s}}{1+\frac{K}{s}(G(s)+d)} \\
& \triangleq \frac{G(s) \bar{C}(s)}{1-G(s) \bar{C}(s)},
\end{aligned}
$$

where

$$
\bar{C}(s) \triangleq \frac{-K}{s+K d} .
$$

In other words the whole scheme can be implemented in a standard feedback setup with $C(s)$ replaced by $\bar{C}(s)$. As it the case of the plant (1) the controller also satisfies the negative imaginary property, for $K<0$. The following theorem in [12] guarantees the closed loop stability of the scheme

Theorem: Given two stable transfer functions $P(s)$ and $Q(s)$, such that both being negative imaginary with 
atleast one of them being strictly negative imaginary, and $P(\infty) Q(\infty)=0$, then the positive feedback connection of $P(s)$ and $Q(s)$ is internally stable if and only if eigenvalues of the matrix $P(0) Q(0)$ are strictly less than one.

Proof: Refer to [12].

It is apparent that $G(s),(1)$, and $\bar{C}(s),(8)$ are strictly negative imaginary and $G(\infty) \bar{C}(\infty)=0$. As the controller gain is positive $K<0$, for the controller to be stable $K d>$ 0 , which implies $d<0$. Eigen value condition implies that

$$
\frac{\Gamma}{\omega_{p}^{2} d}<1
$$

has to hold for closed loop stability. As $d<0$, (9) always holds.

Note that the static gain of (6) is given by

$$
\left|G^{(c l)}(0)\right|=\left|\frac{\Gamma}{d\left(\omega_{p}^{2}+\frac{\Gamma}{d}\right)}\right| .
$$

This implies that the static gain would be equal to one, or 0 $\mathrm{dB}$, if $d=-\frac{2 \Gamma}{\omega_{p}^{2}}$.

\section{Actuation of A Piezoelectric tube}

A piezoelectric tube is a thin-walled cylindrical tube made of piezoelectric material. The inner and outer walls of the tube are coated with a layer of silver. The silver coating acts as the electrodes of the piezoelectric tube. The outer electrode of the piezoelectric tube scanner is axially quartered into four equal sections. A pair of opposite quartered electrodes are referred to as the $x-x$ electrodes and the other pair is referred to as the $y-y$ electrodes. See Figure 2, good illustrations of the tube can be found in [4], [6], [8].

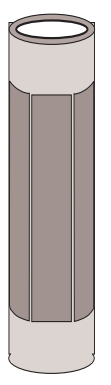

(a)

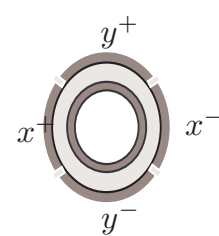

(b)
Fig. 2. Illustration of the piezoelectric tube, (a) side view and (b) top view.

\section{A. Experimental setup}

In the experimental setup used here, a piezoelectric tube is held upright with its bottom glued to a rigid surface. A small aluminum cube is bonded to the upper end of the tube. This cube represents the seat where the materials that need to be scanned are placed. The head of an ADE Technologies 4810 capacitive sensor is placed in close proximity to the face of aluminum cube along the $x$ axis, see Figure 3 . The inner electrode of the piezoelectric tube is grounded. An electrode from the $x-x$ pair, referred as the $x^{+}$electrode, is chosen as the input end of the piezoelectric tube. The whole setup consisting of the piezoelectric tube with the bonded aluminum cube and the heads of the capacitive sensors, is placed in a specially constructed cylindrical enclosure, refer to Figure 4. The cylindrical enclosure protects the experimental setup from external noise.

As mentioned in the introduction the goal is to actuate this piezoelectric tube in a raster pattern. A desired trajectory for the piezoelectric tube would be to repeatedly trace straight lines back and forth in the $x$ direction, while slowly increasing its position in the $y$ direction. A common practice to track such a trajectory is to apply a triangular waveform to the $x^{+}$electrode and a "very slowly" increasing ramp signal to the $y^{+}$(one of the electrodes of the $y-y$ pair, see Figure 2). In fact, to have a good scan of the surface the changes in $y$ direction must be "quasi-static" with respect to the changes in the $x$ direction. Normally for illustration purposes the slowly varying ramp in the $y^{+}$electrode is either replaced by a DC signal or assumed to be earthed or open circuited, see [1], [9], [14]. Here, it is earthed.

When a signal is applied at the electrode $x^{+}$, the piezoelectric tube deforms causing a change in the capacitance between the aluminum cube and the head of the capacitive sensor. The change in the capacitance is used by the capacitive sensor to measure the distance between its head and the aluminum cube. This distance, denoted by $D(t)$, is also recorded as an output. In summary, the piezoelectric tube is modeled as a SISO system with the input being the signal applied at the $x^{+}$electrode and the output being the capacitive sensor measurement $D(t)$.

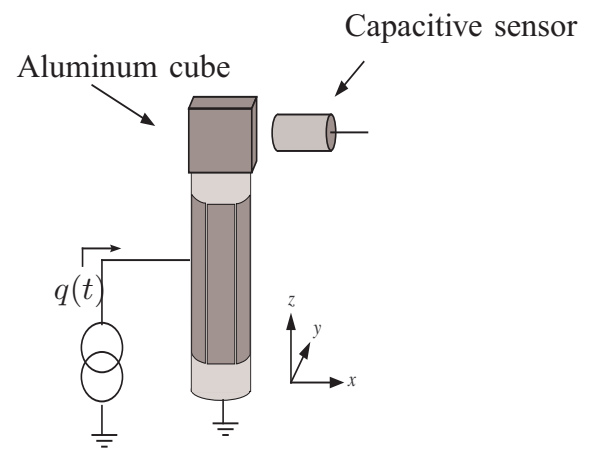

Fig. 3. Schematic Diagram of the experimental setup.

In this paper charge signals $q(t)$, or signals obtained by modulating charges instead of voltages, are used as inputs to avoid hysteresis. In Figures 5 and 6 responses recorded by the capacitive sensor to sinusoidal waveform inputs $v_{1}(t)$ and $q_{1}(t)$, respectively, with a frequency of $5 \mathrm{~Hz}$ are plotted. It can be noted that $v_{1}(t)$ has amplitude of about 62 Volts and $q_{1}(t)$ has an amplitude of $4500 \mathrm{nC}$. More importantly it can be noted that the response to the charge signal is linear while the response recorded for the voltage signal, $v_{1}(t)$ is 


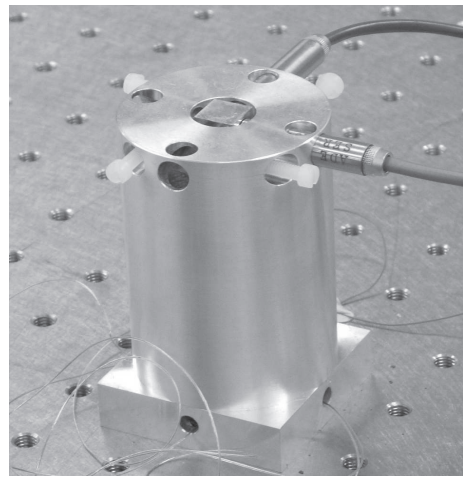

Fig. 4. The piezoelectric tube mounted inside an aluminum shield. The $\mathrm{x}$-axis capacitive sensor is shown secured at right angles to a cube mounted onto the tube tip. And the y-axis capacitive sensor is secured at right angles to the perpendicular face of the aluminum cube.

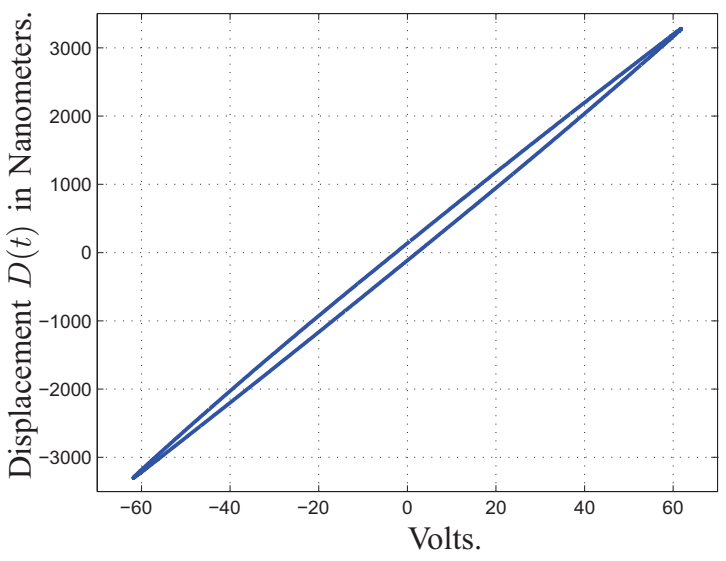

Fig. 5. Response $D(t)$ recorded by the capacitive sensor to a sinusoida input of $5 \mathrm{~Hz}$ frequency and $62 \mathrm{~V}$ amplitude at the $x^{+}$electrode.

non-linear.

\section{B. Identification and Control.}

Using the charge amplifier swept sine waves are applied as input at the $x^{+}$electrode of the piezoelectric tube. Application of the swept sine input causes the tube to bend. The input $q(t)$, applied the $x^{+}$electrode and the corresponding Capacitive sensor output $D(t)$ are fed into a Spectrum Analyzer, which computes the frequency response function (FRF) $G_{d q}(\mathrm{i} \omega)=\frac{D(\mathrm{i} \omega)}{q(\mathrm{i} \omega)}$, where $D(\mathrm{i} \omega)$ and $q(\mathrm{i} \omega)$ are the Fourier transform of $D(t)$ and $q(t)$ respectively, a set of desired frequency points. The computed FRF is plotted in Figure 7. The following model,

$$
G_{d q}(s)=\frac{\Gamma}{s^{2}+2 \sigma_{p} \omega_{p} s+\omega_{p}^{2}},
$$

with the parameters tabulated in Table I, was fit for the FRF data, see Figure 7. It is apparent that the model captures the magnitude response, with good accuracy, but not the phase response. The phase response of the system suggests a delay

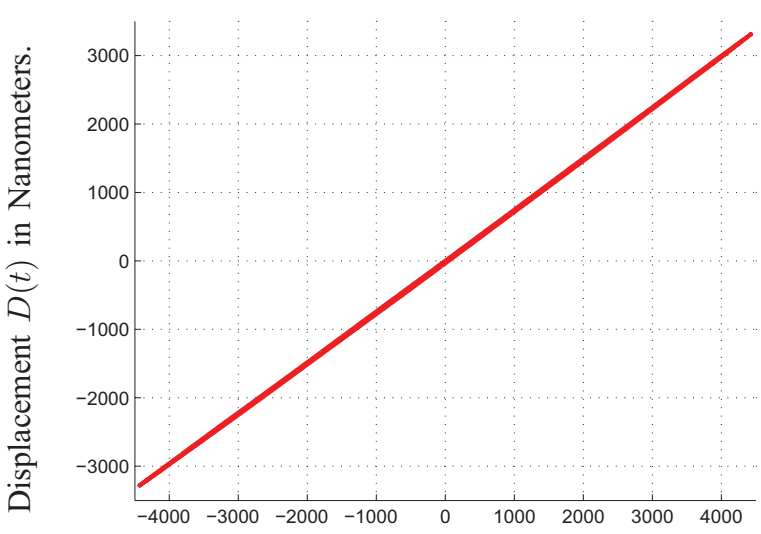

Fig. 6. Response $D(t)$ recorded by the capacitive sensor to a sinusoidal input of $5 \mathrm{~Hz}$ frequency and $4500 \mathrm{nC}$ amplitude at the $x^{+}$electrode.

in the system response. This delay is due to the presence of a zero between the first and the second resonance (the second resonance is not seen in Figure 7, as it is out of the bandwidth of interest), which cannot be captured using a second order model.

\begin{tabular}{|l|l|}
\hline Name & Value \\
\hline$\Gamma$ & $4.6079 \times 10^{5}$ \\
$2 \sigma_{p} \omega_{p}$ & 75.3 \\
$\omega^{2}$ & $2.733 \times 10^{7}$ \\
\hline
\end{tabular}

TABLE I

EMPIRICAL TRANSFER FUNCTION PARAMETERS

As mentioned earlier, to design the control scheme, $d=$ $-\frac{2 \Gamma}{\omega_{p}^{2}}$ is added to obtain

$$
\bar{G}_{d q}(s)=\frac{\Gamma}{s^{2}+2 \sigma_{p} \omega_{p} s+\omega_{p}^{2}}+d .
$$

Note that for the chosen value of $d$ the closed-loop system (6) will have an unit static gain. In Figure 8, the root locus of $\frac{\bar{G}_{d q}(s)}{s}$, or alternatively the locus of the poles of $r(s)=$ $s\left(s^{2}+2 \sigma_{p} \omega_{p} s+\omega_{p}^{2}\right)+K d\left(s^{2}+2 \sigma_{p} \omega_{p} s+\omega_{p}^{2}+\frac{\Gamma}{d}\right)$, for $K<0$, is plotted. Here, the gain of the integral controller is set $K=-10^{5}$. In Figure 9 the magnitude response of $G^{(c l)}(\mathrm{i} \omega)$, (6), is plotted along with experimentally determined magnitude response. It can be observed from the plot that the experimentally determined magnitude response matches predicted magnitude response. Here a dSPACE 1103 PPC controller board is used for the real time implementation of the feed through term $d$ and the integral controller. A sampling frequency of $20 \mathrm{KHz}$ was used to avoid aliasing. Simulink was used to download the feed through term and the controller in to the dSPACE board.

In order to actuate the piezoelectric tube in a raster pattern, an input of the form

$$
u(t) \triangleq \sum_{k=1}^{\infty} \frac{a_{k}}{\left|G_{d q}^{(c l)}\left(\mathrm{i} \omega_{k}\right)\right|} \sin \left(\omega_{k} t-\phi_{k}\right),
$$



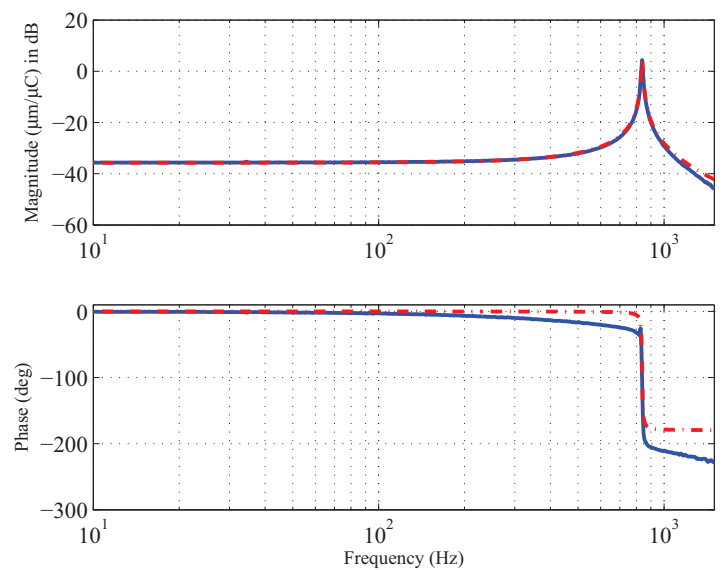

Fig. 7. Magnitude plots of the data (solid) along with the corresponding parametric model (dashed dots)

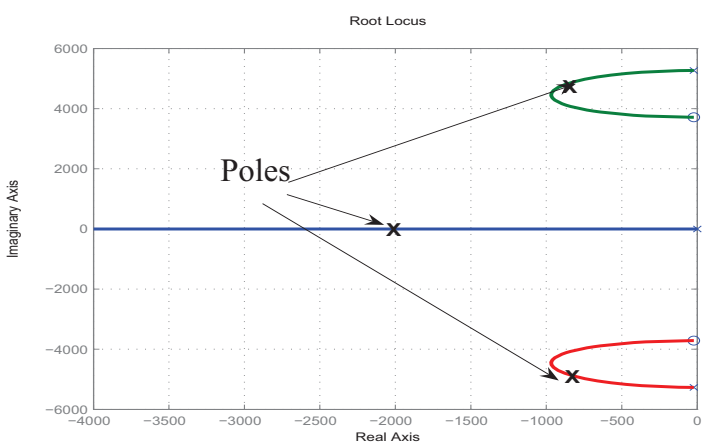

Fig. 8. Root locus of the plot of $\frac{\bar{G}_{d q}(s)}{s}$ for $K<0$. $\mathbf{X}$ denotes the closed-loop poles of $G^{(c l)}(s)$ for a gain of $K=-10^{5}$.
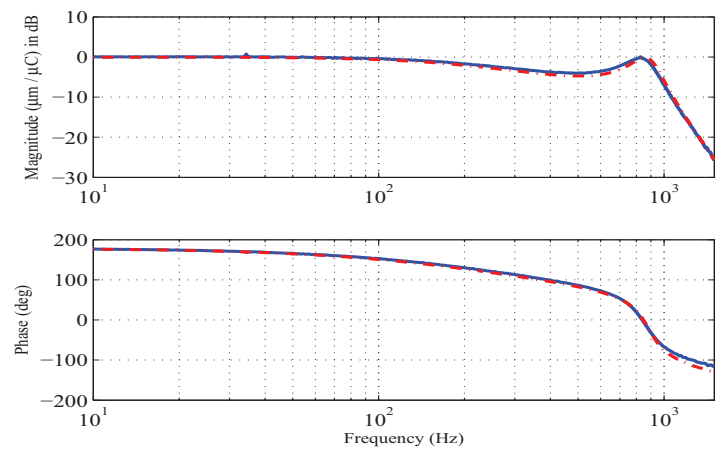

Fig. 9. Predicted (solid) and the experimentally determined (dashed) magnitude responses of the closed-loop system. where $a_{k}$ and $\omega_{k}$ are such that

$$
f_{d}(t) \triangleq \sum_{k=1}^{\infty} a_{k} \sin \left(\omega_{k} t\right)
$$

is the desired triangular waveform output $D(t)$ at the capacitive sensor in the Fourier series form and

$$
\phi_{k} \triangleq \arg G_{d q}^{(c l)}\left(\mathrm{i} \omega_{k}\right),
$$

is applied the $x^{+}$electrode. It is easy to see that applying $u(t)$ to closed-loop plant should give a triangular waveform, provided $G^{(c l)}(\mathrm{i} \omega)$ models the frequency response of the closed-loop plant with reasonable accuracy. It is apparent from Figure 9 that closed-loop model $G_{d q}^{(c l)}(s)$, (6), fits the frequency response of the closed-loop plant with reasonable accuracy. In Figures 10, 11 and 12, the capacitive sensor response $D(t)$ recorded for inputs of the form $u(t)$ with $f_{d}(t)$ being triangular waveforms of $10 \mathrm{~Hz}, 40 \mathrm{~Hz}$ and $80 \mathrm{~Hz}$ respectively. It can be observed that the capacitive sensor responses to inputs $u(t)$, with $10 \mathrm{~Hz}, 40 \mathrm{~Hz}$ and $80 \mathrm{~Hz}, f_{d}(t)$, appear triangular.

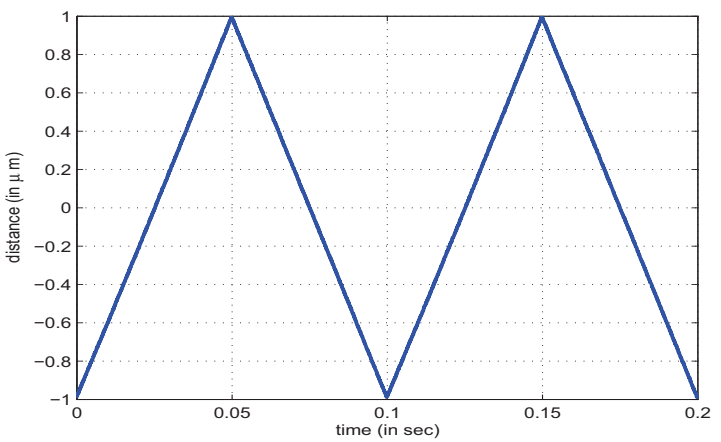

Fig. 10. Response $d_{x}$ recorded by the capacitive sensor for an input $u(t)$ with $f_{d}(t)$ being a $10 \mathrm{~Hz}$ triangular waveform.

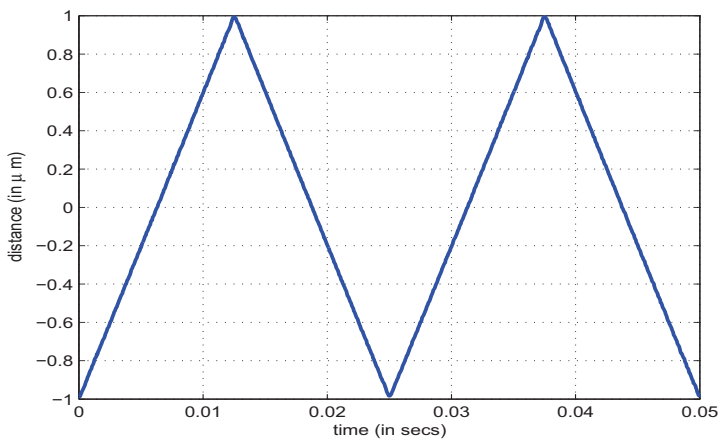

Fig. 11. Response $d_{x}$ recorded by the capacitive sensor for an input $u(t)$ with $f_{d}(t)$ being a $40 \mathrm{~Hz}$ triangular waveform.

In the following the root mean squared errors (RMS) in the capacitive sensor outputs, plotted in Figures 10, 11 and 


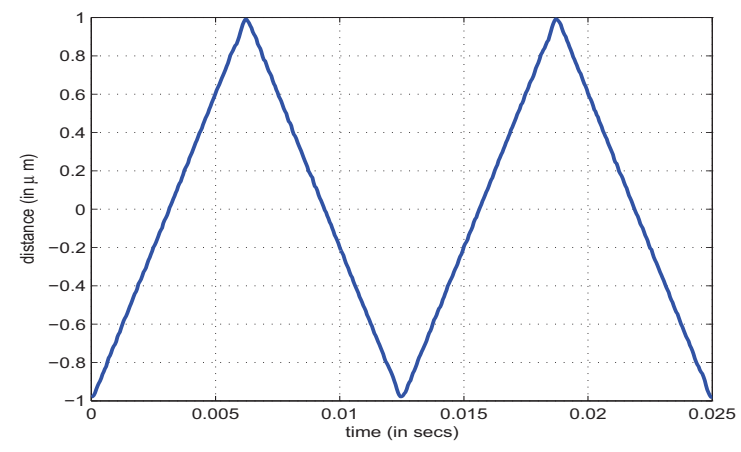

Fig. 12. Response $d_{x}$ recorded by the capacitive sensor for an input $u(t)$ with $f_{d}(t)$ being a $80 \mathrm{~Hz}$ triangular waveform.

12, are estimated using the approximation

$$
\begin{aligned}
\epsilon_{R M S} & \triangleq \sqrt{\frac{1}{T} \int_{0}^{T}\left[f_{d}(t)-f_{o}(t)\right]^{2} d t} \\
& \approx \sqrt{\frac{1}{L} \sum_{k=0}^{L-1}\left[f_{d}(k h)-f_{o}(k h)\right]^{2}} .
\end{aligned}
$$

In (16), $f_{d}(t)$ denotes the desired triangular waveform, $f_{o}(t)$ denotes the capacitive sensor output, $T$ denotes the period of the desired triangular waveform and $h$ denotes the sampling rate. Note that, the desired triangular waveforms, $f_{d}(t)$ 's, corresponding capacitive sensor outputs, $f_{o}(t)$ 's, plotted in Figures 10, 11 and 12 have an amplitude of $1 \mu \mathrm{m}$ with fundamental frequencies being $10 \mathrm{~Hz}, 40 \mathrm{~Hz}$ and $80 \mathrm{~Hz}$ respectively. The estimates of the $\epsilon_{R M S}$, with $h=5 \times 10^{-5}$ sec, for the $10 \mathrm{~Hz}, 40 \mathrm{~Hz}$ and $80 \mathrm{~Hz}$ triangular waveforms were found to be $4.9 \mathrm{~nm}, 9 \mathrm{~m}$ and $15.6 \mathrm{~nm}$ respectively. Not surprisingly, the root mean squared error increases with the increasing frequency of scan. Since high frequency triangular waveforms excite the unmodeld higher order harmonics. It is general practice to consider only $80 \%$ of the scan area. In other words $20 \%$ of the data around the corners, i.e., $20 \%$ data close to the peaks in Figures 10, 11 and 12, are discarded, since these regions correspond to the higher hormonics which excite the unmodeled dyanamics. The $\epsilon_{R M S}$ estimates of the $80 \%$ scans, for $10 \mathrm{~Hz}, 40 \mathrm{~Hz}$ and $80 \mathrm{~Hz}$ triangular waveforms, were found to be $4.7 \mathrm{~nm}, 7.5 \mathrm{~nm}$ and $12.5 \mathrm{~nm}$ respectively.

\section{CONCLUSION}

In this paper a piezoelectric tube of the type used in STMs and AFMs was considered. The objective was to actuate this tube at high scanning frequencies with nano-scale precision. It was noted that the main impediment to perform fast actuation is the presence of a resonant mode. This resonant mode was damped by approximating the tube dynamics by a model satisfying the negative imaginary property, and designing a suitable IRC controller in the feedback. Damping of the resonance mode enabled vibration free actuations with scanning frequencies comparable to $0.1 f_{r}$, where $f_{r}$ is the resonance frequency of the tube. The RMS errors obtained for actuation with scanning frequencies $10 \mathrm{~Hz}, 40 \mathrm{~Hz}$ and $80 \mathrm{~Hz}$ confirm that the scans are of nano-scale precision.

\section{ACKNOWLEDGEMENTS}

The experimental setup, including the charge drive electronics, used here was designed and constructed by Dr. A. J. Fleming.

\section{REFERENCES}

[1] A. Daniele, S. Salapaka, M. V. Salapaka and M. Daleh. Piezoelectric Tubes for Atomic Force Microscopes: Design of Lateral Sensors, Identification and Control. in Proc. of the American Control Conference, San Diego, California, pages 253-257, 1999.

[2] P. K. Hansma et al. High-speed atomic force microscopy. Science, 314(3799):601-602, 2006.

[3] A. J. Fleming and S. O. R. Moheimani. Precision Current and Charge Amplifiers for Driving High Capacitive Piezoelectric Loads. Electronic Letters, 39(3):282-284, Feb 2003.

[4] A. J. Fleming and S. O. R. Moheimani. Sensorless Vibration Supression and Scan Compensation for Piezoelectric Tube Nanopositioners. IEEE Transactions on Control Systems Technology, 14(1):33-44, Jan 2006.

[5] S. S. Aphale, A. J. Fleming, and S. O. R. Moheimani. Integral resonant control of collocated smart structures. Smart Materials and Structures, $16: 439-446,2007$

[6] B. Bhushan Ed. Springer Handbook of Nanotechnology. SpringerVerlag, Heidelberg, Germany, 2004.

[7] D. Hughes and J. T. Wen. Perisach Modeling and Compensation for Smart Material Hysteresis. in Active Materials and Smart Structures, Proc. of SPIE, pages 50-64, 1994

[8] S. Devasia, E. Eleftheriou, and S. O. R. Moheimani. A survey of control issues in nanopositioning. IEEE Transactions on Control Systems Technology, 15(5):802-823, September 2007.

[9] G. Schitter and A. Stemmer. Identification and Open-Loop Tracking Control of a Piezoelectric Tube Scanner for High-Speed Scanning Probe Microscopy. IEEE Transactions on Control Systems Technology, 12(3):449-454, 2004.

[10] K. K. Leang and S. Devasia. Hysteresis, Creep and Vibration Compensation for Piezoactuators: Feedback and Feedforward Control. Proc. of 2nd IFAC Conference on Mechatronic Systems Berkeley California, USA, pages 283-289, 2002.

[11] H. Kaizuka and B. Siu. simple way to reduce hysteresis and creep when using piezoelectric actuators. Japan Journal of Applied Physics, Part 2 - Letters, 27(5):773-776, May 1988.

[12] Alexander Lanzon and Ian R. Petersen. Stability robustness of a feedback interconnection of systems with negative imaginary frequency response. IEEE Transactions on Automatic Control, 53(4):1042-1046, 2008.

[13] I. Mayergoyz. Mathematical Models of Hysteresis and their Applications. Elsevier Science, New York, USA, 2003.

[14] N. Tamer and M. Daleh. Feedback Control of Piezoelectric Tube Scanners. in Proc. of the 33rd Conference on Decision and Control, Lake Buena Vista, Florida, pages 1826-1831, 1994.

[15] C. V. Newcomb and I. Flinn. Improving the linearity of piezoelectric ceramic actuators. IEE Electronics Letters, 18(11):442-443, May 1982.

[16] O. M. El Rifai and K. Youcef-Toumi. Coupling of Piezoelectric Tube Scanning in Scanning Probe Microscopes. in Proc. of the American Control Conference, Arlington, Virginia, pages 3251-3255, 2001.

[17] R. Venkataraman and P. S. Krishnaprasad. Approximate Inversion of Hysteresis: Theory and Numerical Results. Proc. of 39th IEEE Conference on Decision and Control, pages 4448-4454, 2000.

[18] S. Salapaka and A. Sebastian. Control of Nanopositioning Device. in Proc. of the 42nd IEEE Conference on Decision and Control, Maui, Hawaii, pages 2644-2648, 2003.

[19] M. E. Taylor. Dynamics of Piezoelectric Tube Scanners for Scanning Probe Microscopy. Rev. Sci. Instruments, 64(1):154-158, 1993. 